\title{
Prospective implementation of algorithmic patient selection for gastrostomy tube placement consultations: a pre- and post-intervention analysis
}

This article was published in the following Dove Press journal:

Clinical and Experimental Gastroenterology

Joseph J Cano'

Aaron $\mathrm{P}$ Thrift ${ }^{2,3}$

Hashem B El-Serag ${ }^{1,4}$

'Section of Gastroenterology and Hepatology, Department of Medicine, Baylor College of Medicine, Houston, TX, USA; ${ }^{2}$ Section of Epidemiology and Population Sciences, Department of Medicine, Baylor College of Medicine, Houston, TX, USA; ${ }^{3}$ Dan L Duncan Comprehensive Cancer Center, Baylor College of Medicine, Houston, TX, USA; ${ }^{4}$ Department of Medicine, Houston VA HSR\&D Center for Innovations in Quality, Effectiveness and Safety, Michael E. DeBakey Veterans Affairs Medical Center, Houston, TX, USA
Correspondence: Hashem B El-Serag Department of Internal Medicine Gastroenterology, Baylor College of Medicine, Michael E. DeBakey Veterans Affairs Medical Center, 7200 Cambridge Street, Suite A08.I I2, MS: BCM620,

Houston, TX, USA

Tel +l 8327224406

Email hasheme@bcm.edu
Background: Studies have shown high but variable mortality following gastrostomy tube (GT) placement. There are no standard practice guidelines for GT placement.

Aim: To evaluate if implementation of patient selection and prognosis algorithms for GT insertion has favorable effects on patient outcomes.

Methods: This was a pre-, post-cohort analysis in a Veterans Affairs hospital. We implemented a patient selection algorithm aided by the Sheffield Gastrostomy Scoring System (SGSS) in July 2015. We reviewed all referrals to the inpatient service for a GT between July 2014 and June 2016 (pre-, post- implementation), and collected albumin and SGSS at time of consultation, date of GT insertion, and outcomes including vital status and albumin 30 days post-consultation. Patients were followed until 30 October 2016. We compared outcomes pre- and post-implementation.

Results: A total of 126 referrals were reviewed, 68 pre- and 58 post-algorithm implementation. Seventy-seven GTs were placed; 58 (75.3\%) fulfilled the algorithm-appropriate indications. The mean SGSS was significantly lower among patients in whom GT was placed for algorithmappropriate indications 2.03 ( $\mathrm{SD}=0.86)$ vs inappropriate indications $(2.59, \mathrm{SD}=0.63 ; p<0.001)$. Sixty-five $(51.6 \%)$ patients died by conclusion of study. Thirty day mortality after GT placement was $26.2 \%$ (post- $(22.4 \%)$ vs pre- $(29.4 \%))$. Changes in serum albumin at day 30 was nonsignificant.

Conclusion The use of algorithm guidance by the prospective use of the SGSS was associated with a higher likelihood of GT placement both overall and for algorithm-appropriate indications. Keywords: quality, improvement, feeding tube, PEG tube, nutrition, ethics, outcomes

\section{Introduction}

Gastrostomy tube (GT) placement, including percutaneous endoscopic gastrostomy $(\mathrm{PEG})$, is a common practice in patients requiring enteral nutrition due to a variety of conditions that interfere with oral intake. Accepted indications in which patients may benefit from GT placement include esophageal obstruction (eg, head, neck, and esophageal malignancies), and dysphagia associated with neurological disorders (eg, cerebral vascular accident (CVA), amyotrophic lateral sclerosis (ALS), and Parkinson's disease). ${ }^{1}$

However, depending on the etiology of reduced oral intake and the consequences of GT placement, some patients may not benefit or may even be harmed 
by GT placement. ${ }^{1}$ GTs are sometimes placed with unrealistic expectations of benefit (eg, advanced dementia, or reduced risk of aspiration or pressure wounds), to facilitate hospital discharge or transfer to nursing home facilities, or due to a sense of obligation to provide nutrition and hydration. ${ }^{1,2}$ GT placement in these instances is not likely to improve the patients' outcomes. In a cohort of 7,369 veterans who underwent PEG placement between 1989 and 1992, the median survival following PEG was only 7.5 months with an in-hospital mortality rate of $23.5 \%{ }^{3}$ The high mortality associated with GT placement has been attributed to poor premorbid state and to a lesser extent procedural factors. ${ }^{2,4,5}$

There are no standard practice guidelines for GT placement, including PEG. ${ }^{1}$ Often providers use the "gut feeling" test to assess appropriateness for GT placement. However, studies have shown that prediction of prognosis by clinical judgement may be inaccurate compared with a scoring system. ${ }^{5}$ Therefore, there have been several attempts to improve the selection of patients for GT placement, and to assist patients, families, and providers in making a rational decision for or against GT. Rabeneck et al, developed a decision making algorithm for recommending PEG placement based on offering a benefit to the patient, in terms of a positive effect on a physiologic parameter (nutritional status), or a positive effect on quality of life. ${ }^{1,3}$ In addition, the Sheffield Gastrostomy Scoring System (SGSS) is a validated risk assessment tool to predict 30-day mortality for PEG insertion based on age and serum albumin levels; it was derived in a cohort of hospitalized patients having a new PEG inserted. ${ }^{5-7}$ Composite SGSS scores of 0-3 corresponded to 30 -day mortality rates of $0 \%, 7 \%, 21.3 \%$, and $37.3 \%$, respectively. ${ }^{5-7}$

It is unclear however, if the implementation of an algorithmic approach combined with the use of the SGSS to guide patient selection for GT placement would have favorable effects on patient outcomes in practice. We therefore performed a pre-, post-quality improvement initiative to determine the effect of a GT patient selection protocol combined with the SGSS on objective outcomes (eg, in-hospital mortality, 30-day mortality, serum albumin).

\section{Material and methods}

We conducted a pre-, post-intervention cohort analysis of all first time inpatient GT consultations at the Michael E. DeBakey Veterans Affairs (VA) Medical Center between
July 2014 and June 2016. This study was approved as a quality improvement work by the Office of Research Oversight at the Michael E. DeBakey VA Medical Center; patient consent to review their medical records was not required. In the pre-implementation period between July 2014 and June 2015 (prior to implementation of GT patient selection algorithm), we retrospectively reviewed patients who had a consultation for a first time GT to evaluate if they would have fulfilled our appropriate patient selection algorithm. In the post-intervention period between July 2015 and June 2016, all consults were evaluated according to a GT patient selection algorithm (Table 1) aided by the SGSS. The algorithm describes recommendations on whether or not to offer GT, and the method of placing GT (Table 1). The GT indication, recommendation, SGSS, and patient disposition were prospectively recorded.

In all pre- and post-patients, we extracted data from the VA electronic medical records on age, date of GT consultation, reason for GT consultation, serum albumin level and SGSS at time of consultation, date of GT placement (if placed), reason if deferred, and the method of GT insertion (PEG, interventional radiology, surgery). Patients were followed until death or last patient encounter (through 31 October 2016). We collected data on outcomes including in-hospital death at time of GT consult/placement, vital status as of 31 October 2016, GT removal, and serum albumin 30 days post-GT placement (if placed) or consultation. If a GT was deferred, the reason was documented (patient deferred, patient expired, patient regained ability to swallow, inappropriate indication, and other).

\section{Statistical analysis}

We compared GT indications, patient characteristics, consult recommendation, patient disposition, and outcomes after consults between patients in the pre- and post-implementation periods, and between patients who did and did not have a GT placed within these two era using Student's $t$-test for continuous variables and the chi-squared test for categorical variables. We marked the reasons for GT insertion into algorithm-appropriate vs algorithm-inappropriate indications and compared outcomes between patients considered to have had algorithm-appropriate vs algorithm-inappropriate indications for GT placement. The appropriate indications included CVA, ALS, Parkinson disease, and mechanical obstruction, whereas inappropriate indications included dementia, risk of aspiration, and acute alternation of mental status. We performed all analyses using Stata version 13 (StataCorp LP, College Station, 


\begin{tabular}{|c|c|c|c|c|}
\hline 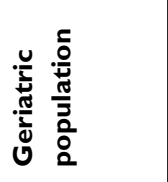 & 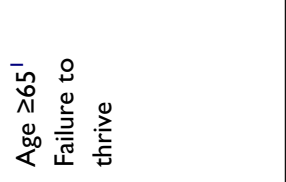 & 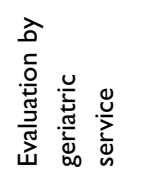 & 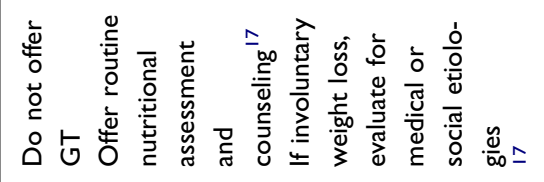 & 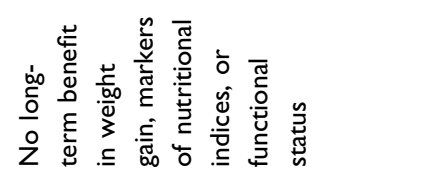 \\
\hline 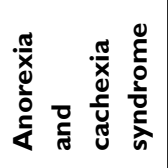 & 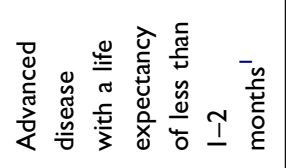 & 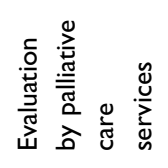 & 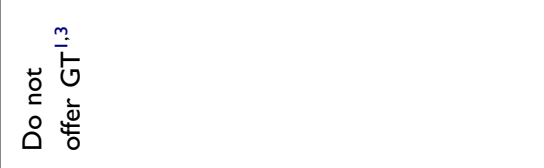 & 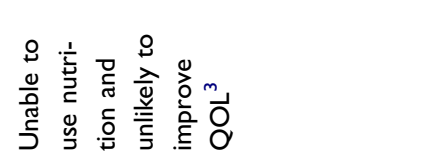 \\
\hline 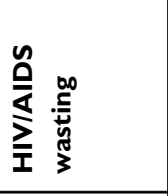 & 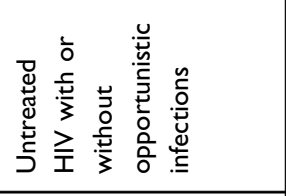 & 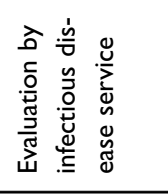 & 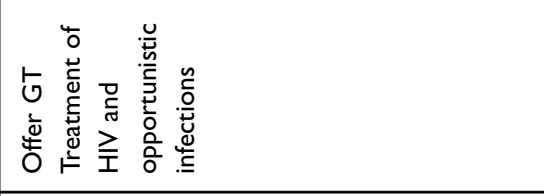 & 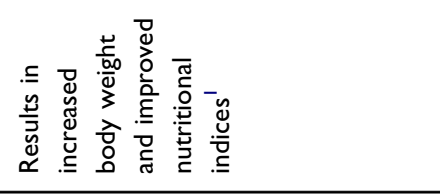 \\
\hline 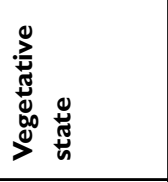 & 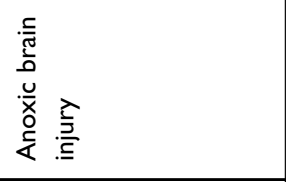 & 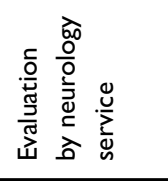 & 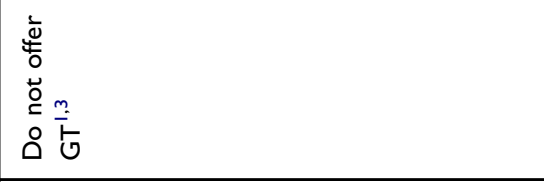 & 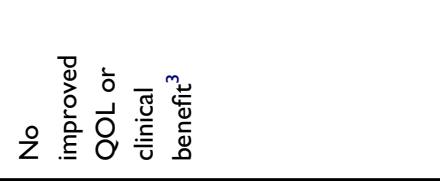 \\
\hline 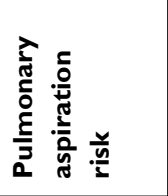 & 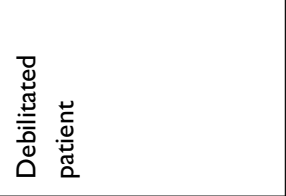 & 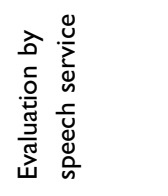 & 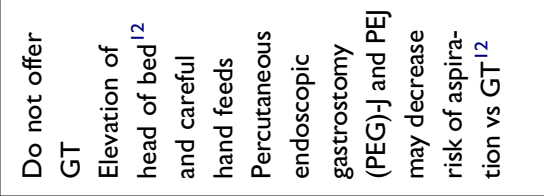 & 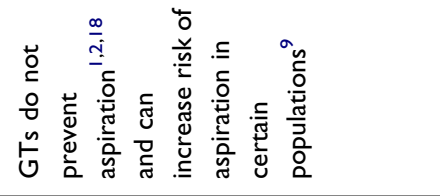 \\
\hline 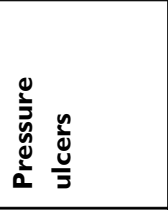 & 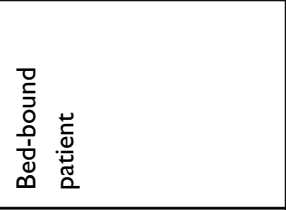 & 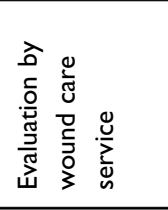 & 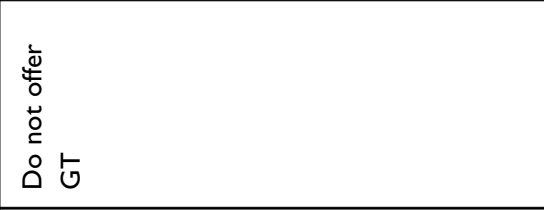 & 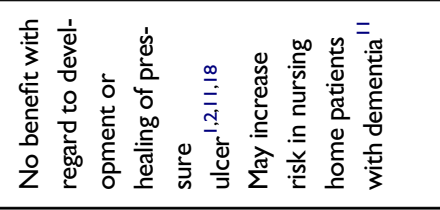 \\
\hline 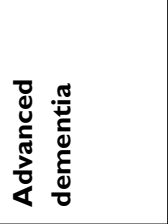 & 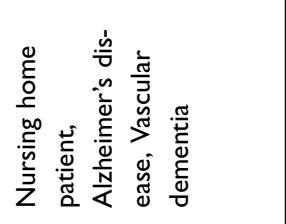 & 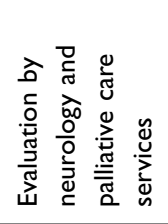 & 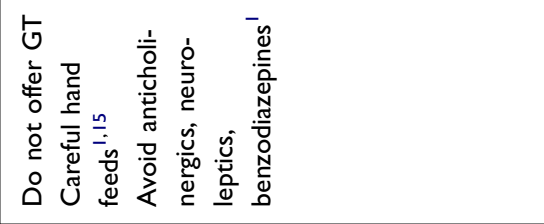 & 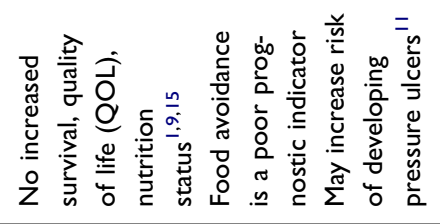 \\
\hline 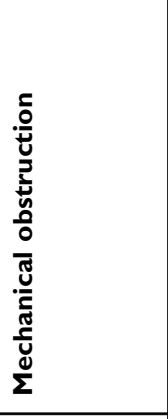 & 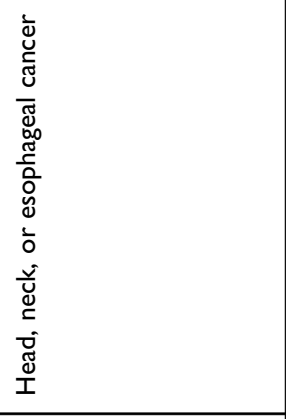 & 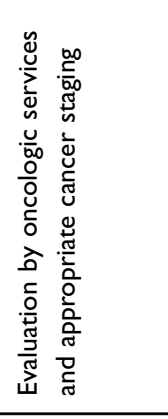 & 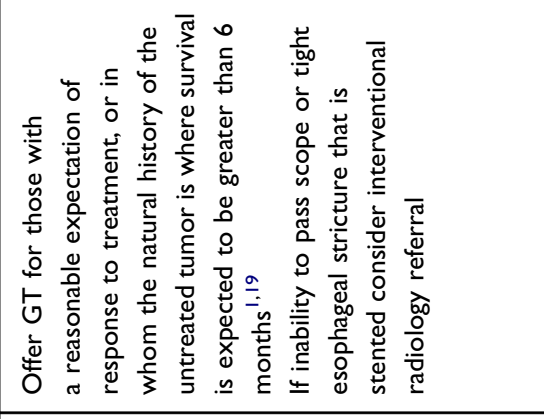 & 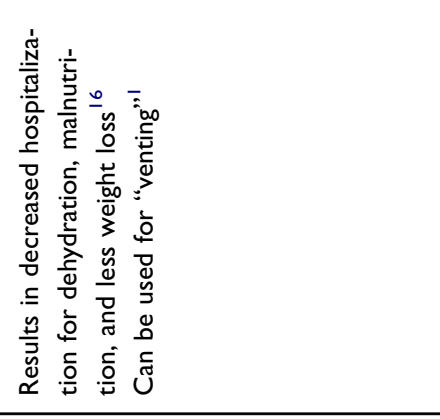 \\
\hline 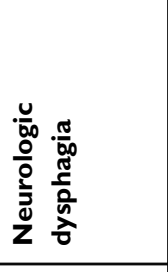 & 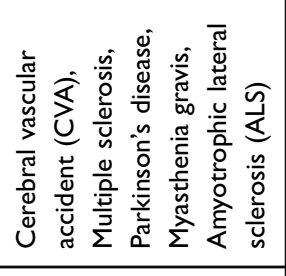 & 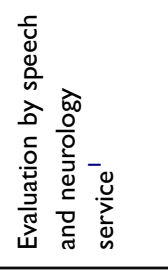 & 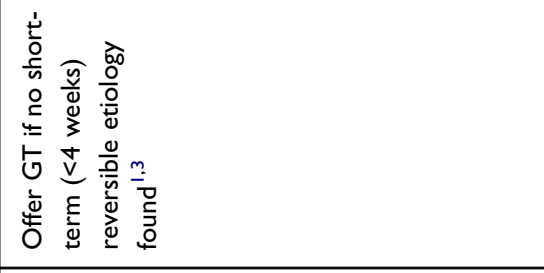 & 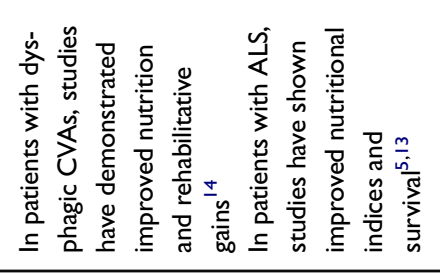 \\
\hline 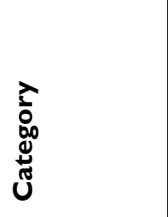 & 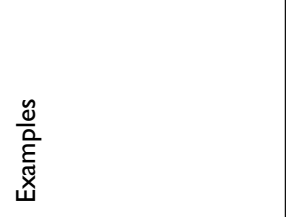 & 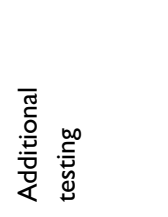 & 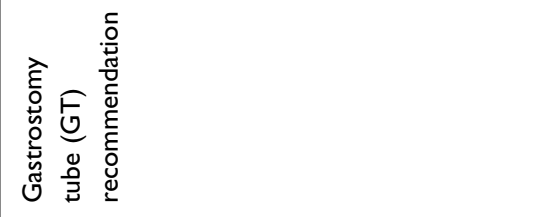 & 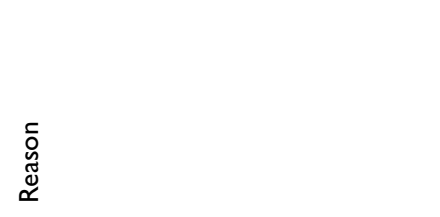 \\
\hline
\end{tabular}


TX, USA). Statistical significance was determined at $\alpha=0.05$, and all $p$-values for statistical significance were two-sided.

\section{Results}

We reviewed a total of 126 GT consultations for unique patients; 68 were made pre- and 58 post-GT algorithm implementation (Table 2). Overall, the mean age of these patients was 70.8 years $(\mathrm{SD}=10.9), 124$ $(98.4 \%)$ were men and $65(51.6 \%)$ were White. There were no significant differences between the demographic or clinical characteristics of the patients in the two eras except for a higher proportion of consults for CVA or neurological disorders in the postimplementation era (Table 2). The proportion of GT consults converted to GT placement, and the proportion of endoscopic GT placement were higher in the postalgorithm era (Table 2). GTs were also more likely to be placed if the consult was for an algorithmappropriate indication, and among patients with lower SGSS. GT placement was also associated with slightly higher serum albumin after 30 days and lower inpatient death than those without GT placed (Table 2).

\section{Indication and method of placed GT}

Of 126 consultations, $73(57.9 \%)$ were for indications deemed to be appropriate in our algorithm. The proportion of algorithm-appropriate GT placements was slightly higher in the post- than pre-implementation period (Table 3). A total

Table 2 Characteristics of the study cohort of 126 patients in whom gastrostomy tube (GT) consults were requested. Comparisons between groups based on GT placement and pre- and post-algorithm eras

\begin{tabular}{|c|c|c|c|c|c|c|}
\hline & $\begin{array}{l}\text { GT not } \\
\text { placed }\end{array}$ & $\begin{array}{l}\text { GT } \\
\text { placed }\end{array}$ & $P$-value & $\begin{array}{l}\text { Pre- } \\
\text { algorithm }\end{array}$ & $\begin{array}{l}\text { Post- } \\
\text { algorithm }\end{array}$ & $P$-value \\
\hline $\mathrm{N}$ & 49 & 77 & & 68 & 58 & \\
\hline Age, Mean (SD) & $74.6(11.0)$ & $68.4(10.2)$ & 0.002 & $70.9(12.3)$ & $70.6(9.1)$ & 0.88 \\
\hline Men & $49(100)$ & $75(97.4)$ & 0.26 & $66(97.1)$ & $58(100)$ & 0.19 \\
\hline Race/Ethnicity & & & 0.28 & & & 0.45 \\
\hline White & $21(42.9)$ & $44(57.1)$ & & $32(47.1)$ & $33(56.9)$ & \\
\hline African American & $23(46.9)$ & $28(36.4)$ & & $31(45.6)$ & $20(34.5)$ & \\
\hline Other & $5(10.2)$ & $5(6.5)$ & & $5(7.3)$ & $5(8.6)$ & \\
\hline Consult for algorithm-approved indication & $15(30.6)$ & $58(75.3)$ & $<0.001$ & $36(52.9)$ & $37(63.8)$ & 0.22 \\
\hline GT placed & $N / A$ & $77(100)$ & - & $37(54.4)$ & $40(69.0)$ & 0.10 \\
\hline GT placed for approved indication & $N / A$ & $58(75.3)$ & - & $26(38.2)$ & $32(55.2)$ & 0.06 \\
\hline GT placed endoscopically & $N / A$ & $56(72.7)$ & - & $26(38.2)$ & $30(5 \mid .7)$ & 0.13 \\
\hline Albumin at consultation, mean (SD) & $2.23(0.64)$ & $2.64(0.68)$ & $0.00 \mathrm{I}$ & $2.49(0.7 I)$ & $2.47(0.67)$ & 0.89 \\
\hline $\begin{array}{l}\text { Albumin at } 30 \text { days post-GT or consultation if GT not } \\
\text { placed (SD) }\end{array}$ & $2.60(0.74)$ & $2.86(0.7 I)$ & 0.18 & $2.82(0.75)$ & $2.76(0.69)$ & 0.77 \\
\hline SGSS, mean (SD) & $2.57(0.65)$ & $2.18(0.84)$ & 0.01 & $2.38(0.8 I)$ & $2.28(0.77)$ & 0.45 \\
\hline Died in same hospitalization & $19(38.8)$ & $6(7.8)$ & $<0.001$ & $15(22.1)$ & $10(17.2)$ & 0.50 \\
\hline 30-day mortality & $22(44.9)$ & II (14.3) & $<0.001$ & $20(29.4)$ & $13(22.4)$ & 0.37 \\
\hline Reason for consult & & & $<0.001$ & & & 0.03 \\
\hline CVA & $5(10.2)$ & $21(27.3)$ & & II (16.2) & $14(24.1)$ & \\
\hline Neurological disorder other than CVA & $2(4.1)$ & $20(26.0)$ & & $8(11.8)$ & $14(24.1)$ & \\
\hline Dementia & $10(20.4)$ & $3(3.9)$ & & $6(8.8)$ & $6(10.3)$ & \\
\hline Altered mental status & $4(8.2)$ & $5(6.5)$ & & $3(4.4)$ & $6(10.3)$ & \\
\hline Cancer & $6(12.2)$ & $12(15.6)$ & & $10(14.7)$ & $7(12.1)$ & \\
\hline Other & $22(44.9)$ & $16(20.8)$ & & $29(42.6)$ & $10(17.2)$ & \\
\hline
\end{tabular}

Abbreviations: SGSS, Sheffield Gastrostomy Scoring System; CVA, cerebral vascular accident. 
Table 3 Comparison of outcomes among four groups based on the timing of gastrostomy tube (GT) consults (pre- and post-algorithm implementation) and their fulfillment of algorithm (- or + ). Total of 126 patients in whom GT consults were requested

\begin{tabular}{|c|c|c|c|c|}
\hline Timing with respect to intervention & & - algorithm $N=53$ & + algorithm $N=73$ & $P$-value \\
\hline Pre- & $\begin{array}{l}\mathrm{N}=68 \\
\mathrm{GT} \text { placed } \\
\text { 30-day mortality } \\
\text { Died same hospitalization } \\
\text { Change in serum albumin }\end{array}$ & $\begin{array}{l}32 \\
11(34.4) \\
12(37.5) \\
9(28.1) \\
0.37(0.84)\end{array}$ & $\begin{array}{l}36 \\
26(72.2) \\
8(22.2) \\
6(16.7) \\
0.17(0.69)\end{array}$ & $\begin{array}{l}0.002 \\
0.17 \\
0.26 \\
0.33\end{array}$ \\
\hline Post- & $\begin{array}{l}\mathrm{N}=58 \\
\mathrm{GT} \text { placed } \\
\text { 30-day mortality } \\
\text { Died same hospitalization } \\
\text { Change in serum albumin }\end{array}$ & $\begin{array}{l}21 \\
8(38.1) \\
7(33.3) \\
6(28.6) \\
0.57(0.89)\end{array}$ & $\begin{array}{l}37 \\
32(86.5) \\
6(16.2) \\
4(10.8) \\
0.13(0.71)\end{array}$ & $\begin{array}{l}<0.001 \\
0.13 \\
0.09 \\
0.12\end{array}$ \\
\hline
\end{tabular}

Note: $P$-value from chi-squared test comparing algorithm-inappropriate and algorithm-appropriate groups.

of 77 GTs were placed (56 PEG, 16 interventional radiology, five surgery); 58 of these (75.3\%) fulfilled the algorithmappropriate indications, while 19 (24.7\%) did not fulfil the indications (Table 3). There was no significant difference in PEG as method of GT placement between the two periods ( $75.0 \%$ vs $70.3 \%)$.

\section{Mortality}

The in-hospital mortality during GT consultation was $19.8 \%$ overall $(7.8 \%$ for those receiving GT, and $38.8 \%$ in those who did not receive GT $(p<0.001))$. After GT placement, the inpatient as well as the 30-day mortality were lower in post-implementation in those who fulfilled algorithms than their counterparts in the preimplementation era, but these differences were not statistically significant (Table 4). Reason for consultation (underlying disease) was not found to be a significant predictor of mortality (Table 2).

\section{SGSS}

At the time of GT consultation the overall mean SGSS was not different between the pre-implementation $(2.38(\mathrm{SD}=0.81))$ and post-implementation periods (2.28 $(\mathrm{SD}=0.77)) ; p=0.45$. The SGSS was $2.20(\mathrm{SD}=0.81)$ in those who were alive at the conclusion of the study and $2.46(\mathrm{SD}=0.75)$ in those who died ( $p=0.06$ ). The SGSS was 2.8 (SD $=0.48)$ in those with inhospital mortality. The SGSS was $2.62(\mathrm{SD}=0.59)$ in those with 30-day mortality. The mean SGSS was significantly lower among patients in whom GT was placed for an algorithm-appropriate indication $(2.11(\mathrm{SD}=0.86))$ than for inappropriate indications $(2.64, \mathrm{SD}=0.56) ; p<0.001$.

\section{Serum albumin}

The mean serum albumin levels were $2.48 \mathrm{~g} / \mathrm{dL}$ ( $\mathrm{SD}=0.69$ ) and $2.79 \mathrm{~g} / \mathrm{dL}(\mathrm{SD}=0.72)$ at 30 days post-consultation or postGT placement, respectively. In patients who had GT placement the mean serum albumin was $2.64 \mathrm{~g} / \mathrm{dL}(\mathrm{SD}=0.68)$ at

Table 4 Comparison of outcomes among four groups based on the timing of gastrostomy tube (GT) consults (pre- and post-algorithm implementation) and their fulfillment of algorithm (-or +). Total of 77 patients in whom GT was placed

\begin{tabular}{|c|c|c|c|c|}
\hline & & $\begin{array}{l}\text { - algorithm } \\
N=19\end{array}$ & $\begin{array}{l}+ \text { algorithm } \\
\mathbf{N}=\mathbf{5 8}\end{array}$ & $P$-value \\
\hline Pre- & $\begin{array}{l}\mathrm{N}=37 \\
\text { 30-day mortality } \\
\text { Died same hospitalization } \\
\text { Change in serum albumin }\end{array}$ & $\begin{array}{l}\text { II } \\
\text { I }(9.1) \\
\text { I }(9.1) \\
0.57(0.73)\end{array}$ & $\begin{array}{l}26 \\
4(15.4) \\
2(7.7) \\
0.12(0.68)\end{array}$ & $\begin{array}{l}0.61 \\
0.89 \\
0.14\end{array}$ \\
\hline Post- & $\begin{array}{l}\mathrm{N}=40 \\
30 \text {-day mortality } \\
\text { Died same hospitalization } \\
\text { Change in serum albumin }\end{array}$ & $\begin{array}{l}8 \\
2(25.0) \\
2(25.0) \\
0.63(0.98)\end{array}$ & $\begin{array}{l}32 \\
4(12.5) \\
1(3.1) \\
0.14(0.74)\end{array}$ & $\begin{array}{l}0.38 \\
0.04 \\
0.17\end{array}$ \\
\hline
\end{tabular}

Note: $P$-value from chi-squared test comparing algorithm-inappropriate and algorithm-appropriate groups. 
time of consultation and $2.86 \mathrm{~g} / \mathrm{dL}(\mathrm{SD}=0.71)$ after 30 days, whereas in those who did not undergo GT placement the mean albumin at time of consultation was $2.23 \mathrm{~g} / \mathrm{dL}(\mathrm{SD}=0.64$; $p=0.001)$ and after 30 days was $2.60 \mathrm{~g} / \mathrm{dL}(\mathrm{SD}=0.74)$ $(p=0.18)$.

\section{GT placed for algorithm-inappropriate indications}

Table 3 shows that patients who fulfilled the algorithm were more likely to receive a GT irrespective of pre- or post-eras, but that the highest proportion of GTs placed according to the algorithm was observed in the postimplementation era $(86.5 \%$ vs $72.2 \%$ in the preimplementation era; $p=0.13)$. Similarly, the proportion of GTs placed for inappropriate indications declined slightly from $11 / 37(29.7 \%)$ in the pre-implementation to $8 / 40$ $(20.0 \%)$ in the post-implementation group (Table 4). Patients in whom GT was placed for algorithminappropriate indications had higher 30-day mortality and significantly higher inpatient mortality than those who had GT placed for algorithm-appropriate indications (Table 4).

\section{Discussion}

The use of a prospectively implemented clinical algorithm guided by the prospective use of the SGSS was associated with a higher likelihood of GT placement both overall and for algorithm-appropriate indications. Moreover, the inhospital as well as 30-day mortality was slightly lower than those seen in the pre-implementation era or those in whom GTs were placed for algorithm-inappropriate indications in the post-implementation era.

Since the performance of an algorithm with or without the SGSS is unknown in clinical practice, we provided the recommendation but did not mandate implementation or reject persistent request for GT placement. This approach allowed us to examine the outcomes of patients in whom GT was placed for an algorithm-inappropriate indication. The findings indicate better outcomes for an algorithmbased approach, although the numbers were too small to achieve statistical significance. The relative ease of application means that this approach could be applied reliably by learners, junior physicians, and non-GI specialists.

The SGSS was developed and validated in a United Kingdom population, and may not have been generalizable to a US population because of the lower 30-day mortality (12.7\%) observed in their cohort compared to nearly $25 \%$ observed in large US studies. ${ }^{8}$ However, in our study, the in-hospital mortality during GT consultation was $19.8 \%$ (and 7.8\% for those receiving GT). While our study did not set out to validate the SGSS, this relatively simple score seems to be associated with the inpatient as well as 30-day mortality irrespective of GT placement.

Our study has a number of limitations. The study population was relatively small, which may have masked the significance of some of the differences, and was almost exclusively men. Additionally, our study consisted of only inpatient consultations where acute illness, older age, lower body mass index, and advanced malignancy are often seen and are associated with worse outcomes with PEG placement. ${ }^{2,4,9,10}$ The generalizability to other populations or to the outpatient setting is unclear.

In addition to using algorithms, imposing a waiting period ranging from 7-60 days after GT consultation has been suggested as a way to improve the selection and outcomes of patients referred for GT. ${ }^{2,4,9,10}$ Abuksis et al, reported that PEG tube insertion 30 days after hospital discharge was associated with up to $40 \%$ lower 30 -day mortality rate than PEG insertion during hospitalization $(P=0.01){ }^{2,9}$ Nasogastric feeding period may be more appropriate in patient groups unlikely to survive beyond 30 days. ${ }^{4,9}$

It may be possible to conduct a randomized controlled study in which referred patients for GT are assigned to an algorithmic approach aided by the SGSS or usual care, however, such study would require careful ethical consideration. $^{2}$ In the meantime we recommend applying an iterative algorithmic approach to provide objective support to health care providers and families involved in this difficult decision making process.

\section{Acknowledgment}

The authors acknowledge Dr Maria Velez's contribution in designing the quality improvement effort.

\section{Disclosure}

The authors report no conflicts of interest in this work.

\section{References}

1. Angus F, Burakoff R. The percutaneous endoscopic gastrostomy tube: medical and ethical issues in placement. Am $J$ Gastroenterol. 2003;98:272-277. doi:10.1111/j.1572-0241.2003.07710.x

2. Arora G, Rockey D, Gupta S. High in-hospital mortality after percutaneous endoscopic gastrostomy: results of a nationwide population-based study. Clin Gastroenterol Hepatol. 2013;11:1437-1444. doi:10.1016/j. cgh.2013.04.011

3. Rabeneck L, McCullough LB, Wray NP. Ethically justified, clinically comprehensive guidelines for percutaneous endoscopic gastrostomy tube placement. Lancet. 1997;349:496-498. 
4. Longcroft-Wheaton G, Marden P, Colleypriest B, Gavin D, Taylor G, Farrant M. Understanding Why Patients Die after Gastrostomy Tube Insertion: A Retrospective Analysis of Mortality. JPEN J Parenter Enteral Nutr. 2009 Jul-Aug; 33(4):375-9. doi: 10.1177/ 0148607108327156.

5. Wilcox C, McClave S. To PEG or not to PEG. Clin Med. 2002;2:250-255. doi:10.7861/clinmedicine.2-3-250

6. Kurien M, Leeds JS, DeLegge MH, et al. Mortality among patients who receive or defer gastrostomies. Clin Gastroenterol Hepatol. 2013;11:1445-1450. doi:10.1016/j.cgh.2013.04.025

7. Leeds JS, McAlindon ME, Grant J, et al. Albumin level and patient age predict outcomes in patients referred for gastrostomy insertion: internal and external validation of a gastrostomy score and comparison with artificial neural networks. Gastrointest Endosc. 2011;74:1033-1039. doi:10.1016/j.gie.2011.07.043

8. Leeds JS, Morley SR, Marr R, et al. Predicting outcomes following gastrostomy insertion using the sheffield gastrostomy score: a prospectively devised scoring system with a validation cohort. Proc Nutr Soc. 2009;68:E12. doi:10.1017/S0029665109001530

9. Udd M, Lindström O, Mustonen H, Bäck L, Halttunen J, Kylänpää L. Assessment of indications for percutaneous endoscopic gastrostomy development of a predictive model. Scand $J$ Gastroenterol. 2015;50:245-252. doi:10.3109/00365521.2014.927914

10. Abuksis G, Mor M, Segal N, et al. Percutaneous endoscopic gastrostomy: high mortality rates in hospitalized patients. $\mathrm{Am}$ J Gastroenterol. 2000;95:0-4. doi:10.1111/j.1572-0241.2000.03381.x

11. Teno JM, Gozalo P, Mitchell SL, Kuo S, Fulton AT, Mor V. Feeding tubes and prevention or healing of pressure ulcers. Arch Intern Med. 2012;172:697-701. doi:10.1001/archinternmed.2012.1200
12. Pearce CB, Duncan HD. Enteral feeding. Nasogastric, nasojejunal, percutaneous endoscopic gastrostomy, or jejunostomy: its indications and limitations. Postgrad Med J. 2002;78:198-204. doi:10.1136/ pmj.78.918.198

13. Langmore SE, Kasarskis EJ, Manca ML, Olney RK. Enteral Tube Feeding for Amyotrophic Lateral Sclerosis/Motor Neuron Disease. Cochrane Data Syst Rev. 2006;(4): CD004030. Review. Update in: Cochrane Database Syst Rev. 2011;(1):CD004030.

14. James A, Kapur K, Hawthorne AB. Long-term outcome of percutaneous endoscopic gastrostomy feeding in patients with dysphagic stroke. Age Ageing. 1998;27:671-676. doi:10.1093/ageing/ 27.6.671

15. American Geriatrics Society Ethics Committee and Clinical Practice and Models of Care Committee. American geriatrics society feeding tubes in advanced dementia position statement. J Am Geriatr Soc. 2014;62:1590-1593. doi:10.1111/jgs.13053

16. Lee JH, Machtay M, Unger LD, et al. Prophylactic gastrostomy tubes in patients undergoing intensive irradiation for cancer of the head and neck. Arch Otolaryngol Head Neck Surg. 1998;124:871-875. doi:10.1001/archotol.124.8.871

17. Reuben DB. Quality indicators for the care of undernutrition in vulnerable elders. J Am Geriatr Soc. 2007;55. doi:10.1111/j.15325415.2007.01353.x

18. Nair S, Hertan H, Pitchumoni CS. Hypoalbuminemia is a poor predictor of survival after percutaneous endoscopic gastrostomy in elderly patients with dementia. Am J Gastroenterol. 2000;95:133-136. doi:10.1111/j.1572-0241.2000.03381.x

19. Finestone HM. Safe feeding methods in stroke patients. Lancet. 2000;355:1662-1663. doi:10.1016/S0140-6736(05)72455-7
Clinical and Experimental Gastroenterology

\section{Publish your work in this journal}

Clinical and Experimental Gastroenterology is an international, peerreviewed, open access, online journal publishing original research, reports, editorials, reviews and commentaries on all aspects of gastroenterology in the clinic and laboratory. This journal is indexed on American Chemical Society's Chemical Abstracts Service (CAS).
The manuscript management system is completely online and includes a very quick and fair peer-review system, which is all easy to use. Visit http://www.dovepress.com/testimonials.php to read real quotes from published authors. 